\title{
VECTOR-VALUED SUMMABILITY METHODS ON \\ A LINEAR NORMED SPACE
}

\author{
L. C. KURTZ AND D. H. TUCKER
}

1. Introductions and definitions. Suppose $X$ and $Y$ are linear normed spaces, $B=B[X, Y]$ is the space of bounded linear transformations from $X$ into $Y$, and $C$ is the space of $X$-valued continuous functions on $0 \leqq t \leqq 1$ with $\|f\|_{C}=\max _{0 \leq t \leq 1}\|f(t)\|_{X}$. An integral representation theorem for the transformations $T \in B[C, Y]$ was recently given by Tucker [8]. This representation theorem includes the classical Riesz representation theorem [6] as a special case. Hildebrandt [4] and Hildebrandt and Schoenberg [5] have shown that the classical Riesz theorem is equivalent to the Hausdorff theorem [3] on convergence-preserving Hausdorff summability methods. In this paper we will discuss summability methods in the general setting of the representation theorem given by Tucker, and show that the representation theorem is equivalent to a general Hausdorff theorem.

We suppose that $Y^{+}$is the weak extension [8] of $Y$.

Definition 1 . The statement that $\mathscr{T}$ is a summability method from $X$ to $Y^{+}$means that $刃(t)$ is a matrix $\left(f_{m n}\right)$ of elements of $B$.

Definition 2. The statement that $\mathfrak{T}$ is a convergence-preserving method means that if $\left\{x_{n}\right\}$ is a convergent sequence of points in $X$, then $y_{m}=\sum_{n=0}^{\infty} f_{m n}\left(x_{n}\right)$ exists in $Y^{+}$for each $m$, and $\left\{y_{m}\right\}$ is a convergent sequence of points in $\mathrm{Y}^{+}$.

Definition 3. If $L$ is a linear transformation from $X$ to $Y^{+}$, the statement that $\mathscr{N}$ is regular relative to $L$ means that $\mathscr{N}$ is convergence preserving and has the property that if $x_{n}$ converges to $x$ in $X$, then $y_{m}$ converges in $Y^{+}$to $L(x)$.

Definition 4 . The statement that a summability method $\mathbb{M}$ is a Hausdorff method generated by the sequence $\left\{\mu_{n}\right\}$ of points in $B$ means that $\mathfrak{T}=\rho \mu \rho$, where $\mu=\operatorname{diag}\left(\mu_{0}, \mu_{1}, \cdots\right)$ and $\rho=\left(\rho_{m n}\right)$ is the differencing matrix given by

$$
\begin{aligned}
& \rho_{m n}=(-1)^{n}\left(\begin{array}{l}
m \\
n
\end{array}\right) \text { if } n \leqq m, \\
& \rho_{m n}=0 \text { if } n>m .
\end{aligned}
$$

A direct computation [3] shows that $\mathfrak{T}$ is a Hausdorff method generated by $\left\{\mu_{n}\right\}$ if and only if $\Re=\left(\lambda_{m n}\right)$, where

\footnotetext{
Presented to the Society, January 24, 1964; received by the editors February 13, 1964.
} 


$$
\begin{aligned}
& \lambda_{m n}=\left(\begin{array}{l}
m \\
n
\end{array}\right) \Delta^{m-n} \mu_{n} \text { if } n \leqq m, \\
& \lambda_{m n}=0 \text { if } n>m .
\end{aligned}
$$

2. Convergence-preserving methods. In this section we seek necessary and sufficient conditions for $\mathfrak{T}$ to be convergence preserving. The resulting theorem includes the classical Toeplitz theorem [3] as a special case. Consider the following conditions.

Condition A. There exists a number $M$ such that if $\left\{x_{n}\right\}$ is a bounded sequence of points in $X$, then $\left\|\sum_{n=0}^{p} f_{m n}\left(x_{n}\right)\right\|_{Y} \leqq M \sup _{n}\left\|x_{n}\right\|_{X}$ for all non-negative integers $p$ and $m$.

Condition B. If $x \in X$, then for each non-negative integer $n, f_{m n}(x)$ converges in $Y^{+}$as $m \rightarrow \infty$. We will call this limit $c_{n}(x)$.

Condition C. If $x \in X$, then $r_{m}(x)=\sum_{n=0}^{\infty} f_{m n}(x)$ converges in $Y^{+}$as $m \rightarrow \infty$. We will call this limit $L(x)$.

It will be shown in the next section that Condition $\mathrm{A}$ implies that the function $r_{m}(x)$ defined in Condition $\mathrm{C}$ exists.

Lemma 1. Condition A implies that the numbers $\left\|f_{m n}\right\|_{B}$ are bounded uniformly in $m$ and $n$.

Proof. Consider an $f_{m n}$. If $\epsilon>0$ there exists $x \in X$ such that $\|x\|_{X}=1$ and $\left\|f_{m n}\right\|_{B} \leqq\left\|f_{m n}(x)\right\|_{Y}+\epsilon$. If a sequence $\left\{x_{j}\right\}$ is defined by $x_{j}=\theta$ if $j \neq n$ and $x_{j}=x$ if $j=n$, then

$$
\left\|f_{m n}(x)\right\|_{Y}=\left\|\sum_{j=0}^{n} f_{m n}\left(x_{j}\right)\right\|_{Y} \leqq M \sup _{j}\left\|x_{j}\right\|_{X}=M .
$$

Thus $\left\|f_{m n}\right\|_{B} \leqq M+\epsilon$, which implies that $\left\|f_{m n}\right\|_{B} \leqq M$.

Lemma 2. Conditions A and B together imply that $c_{n} \in B\left[X, Y^{+}\right]$.

Proof. It is clear from definition that $c_{n}$ is linear. To show that $c_{n}$ is bounded, we consider $y^{*} \in Y^{*}$ where $Y^{*}$ denotes the conjugate space of $Y$. Then

$$
\begin{aligned}
\left\|c_{n}(x)\right\| Y_{Y^{+}} & =\sup _{\|y *\| \leqq 1}\left|\lim _{m \rightarrow \infty} y^{*} f_{m n}(x)\right| \leqq \sup _{\|y *\| \leqq 1} \limsup _{m \rightarrow \infty}\left\|y^{*}\right\|\left\|f_{m n}(x)\right\|_{Y} \\
& \leqq \sup _{m}\left\|f_{m n}\right\|_{B}\|x\|_{X} \leqq M\|x\|_{X}
\end{aligned}
$$

by Lemma 1 .

Lemma 3. Condition A implies that $\sum_{n=0}^{\infty} c_{n}\left(x_{n}\right)$ exists in $Y^{+}$for any bounded sequence $\left\{x_{n}\right\}$ of points in $X$. 
Proof. Suppose $y_{1}^{*}, y_{2}^{*} \in Y^{*}$. Then $y_{1}^{*}$ may be extended to operate on $Y^{+}[8]$, and if $\epsilon_{n}=\operatorname{sgn} y_{1}^{*} c_{n}\left(x_{n}\right)$, we have

$$
\begin{aligned}
\sum_{n=0}^{p}\left|y_{1}^{*} c_{n}\left(x_{n}\right)\right| & =\sum_{n=0}^{p} y_{1}^{*} c_{n}\left(\epsilon_{n} x_{n}\right)=y_{1}^{*} \sum_{n=0}^{p} c_{n}\left(\epsilon_{n} x_{n}\right) \\
& =\left|y_{1}^{*} \sum_{n=0}^{n} c_{n}\left(\epsilon_{n} x_{n}\right)\right| \leqq\left\|y_{1}^{*}\right\| Y^{*}\left\|\sum_{n=0}^{p} c_{n}\left(\epsilon_{n} x_{n}\right)\right\|_{Y_{+}} \\
& =\left\|y_{1}^{*}\right\|_{Y^{*}} \sup _{\left\|\nu_{2}\right\|_{1} \leqq 1}\left|\lim _{m \rightarrow \infty} y_{2}^{*} \sum_{n=0}^{p} f_{m n}\left(\epsilon_{n} x_{n}\right)\right| \\
& \leqq\left\|y_{1}^{*}\right\|_{Y^{*}} \sup _{\left\|y_{2} *\right\| \leqq 1} \limsup _{m \rightarrow \infty}\left\|y_{2}^{*}\right\| Y_{Y^{*}}\left\|\sum_{n=0}^{p} f_{m n}\left(\epsilon_{n} x_{n}\right)\right\|_{Y} \\
& \leqq\left\|y_{1}^{*}\right\|_{Y^{*} M \sup _{n}\left\|x_{n}\right\|_{X} .}
\end{aligned}
$$

It follows that $y_{1}^{*} \sum_{n=0}^{p} c_{n}\left(x_{n}\right)$ converges absolutely, so $\sum_{n=0}^{\infty} c_{n}\left(x_{n}\right)$ exists in $Y^{+}$for each $x \in X$.

Lemma 4. Conditions A and $\mathrm{C}$ together imply that $L \in B\left[X, Y^{+}\right]$.

Proof. $L$ is clearly linear, and the proof that it is bounded is essentially the same as the proof of Lemma 2.

Lemma 5. If $X$ is complete, then the space $\chi$ of convergent sequences $\left\{x_{n}\right\}$ of points in $X$ with $\left\|\left\{x_{n}\right\}\right\|_{X}=\sup _{n}\left\|x_{n}\right\|_{X}$ is complete.

The proof of Lemma 5 is straightforward and is omitted.

We are now prepared to give a characterization of convergencepreserving methods.

THEOREM 1. If $X$ is complete, then $\mathfrak{T}$ is convergence preserving if and only if Conditions A, B, and C hold.

Proof. We first show the sufficiency of Conditions $\mathrm{A}, \mathrm{B}$, and $\mathrm{C}$. Suppose $x_{n} \rightarrow \theta$ in $X$. If $\epsilon>0$, there exists a number $N>0$ such that $\left\|x_{n}\right\|_{X}<\epsilon / 4 M$ and $\left\|\sum_{j=n+1}^{\infty} c_{j}\left(x_{j}\right)\right\|_{Y^{+}}<\epsilon / 4$ if $n>N$. Then

$$
\begin{aligned}
\| y_{m} & -\sum_{j=0}^{\infty} c_{j}\left(x_{j}\right) \|_{Y^{+}} \\
& \leqq\left\|\sum_{j=0}^{n}\left[f_{m j}\left(x_{j}\right)-c_{j}\left(x_{j}\right)\right]\right\|_{Y^{+}}+\left\|\sum_{j=n+1}^{\infty} f_{m j}\left(x_{j}\right)\right\|_{Y^{+}}+\left\|\sum_{j=n+1}^{\infty} c_{j}\left(x_{j}\right)\right\|_{Y^{+}} \\
& \leqq\left\|\sum_{j=0}^{n}\left[f_{m j}\left(x_{j}\right)-c_{j}\left(x_{j}\right)\right]\right\|_{Y^{+}}+M \sup _{j>n}\left\|x_{j}\right\|_{X}+\left\|\sum_{j=n+1}^{\infty} c_{j}\left(x_{j}\right)\right\|_{Y^{+}} .
\end{aligned}
$$

Now we pick $n=n_{0}>N$. By Condition B we may pick $m$ large enough 
so that each term in the first expression is less than $\epsilon / 4 n_{0}$. Combining this with the other estimates given above, we have $\left\|y_{m}-\sum_{j=0}^{\infty} c_{j}\left(x_{j}\right)\right\|_{Y^{+}}$ $\leqq \epsilon n_{0} / 4 n_{0}+M(\epsilon / 4 M)+\epsilon / 4<\epsilon$ if $m$ is sufficiently large. Thus $y_{m}$ converges (to $\sum_{j=0}^{\infty} c_{j}\left(x_{j}\right)$ ) in $Y^{+}$. Now suppose that $x_{n} \rightarrow x \neq \theta$. If $x_{n}^{\prime}$ $=x_{n}-x$, then $x_{n}^{\prime} \rightarrow \theta$ and by the above argument, $y_{m}^{\prime}=\sum_{n=0}^{\infty} f_{m n}\left(x_{n}^{\prime}\right)$ converges in $Y^{+}$to $\sum_{j=0}^{\infty} c_{j}\left(x_{j}^{\prime}\right)$ as $m \rightarrow \infty$. Then $y_{m}=\sum_{n=0}^{\infty} f_{m n}\left(x_{n}\right)$ $=\sum_{n=0}^{\infty} f_{m n}\left(x_{n}^{\prime}+x\right)=\sum_{n=0}^{\infty} f_{m n}\left(x_{n}^{\prime}\right)+\sum_{n=0}^{\infty} f_{m n}(x)=y_{m}^{\prime}+r_{m}(x)$. But this converges in $Y^{+}$to $\sum_{j=0}^{\infty} c_{j}\left(x_{j}^{\prime}\right)+L(x)$. Therefore, $y_{m}$ converges in $Y^{+}$to $\sum_{j=0}^{\infty} c_{j}\left(x_{j}^{\prime}\right)+L(x)=\sum_{j=0}^{\infty} c_{j}\left(x_{j}\right)+\left(L-\sum_{j=0}^{\infty} c_{j}\right)(x)=y$. Hence Conditions A, B, and C are sufficient.

Now suppose that $\mathscr{N}$ is convergence preserving. Suppose $\left\{x_{n}\right\}$ $=\{x\}$, then $x_{n} \rightarrow x$ so $y_{m}=\sum_{n=0}^{\infty} f_{m n}(x)$ converges in $Y^{+}$, i.e., Condition $\mathrm{C}$ is necessary.

If we let $x_{n}=x$ if $n=k$ and $x_{n}=\theta$ if $n \neq k$ so that $x_{n} \rightarrow \theta$, then $y_{m}$ $=\sum_{n=0}^{\infty} f_{m n}\left(x_{n}\right)=f_{m k}(x)$ converges in $Y^{+}$. This gives the necessity of Condition B.

Condition A requires a different approach. We use the fact that $Y^{+}$is imbedded isomorphically and isometrically in $Y^{* *}$ [8]. Define transformations $F_{m p}$ of $\chi$ into $Y^{* *}$ by $F_{m p}\left\{x_{n}\right\}=\sum_{n=0}^{p} f_{m n}\left(x_{n}\right)$, where this is considered as a point in $Y^{* *} . F_{m p}$ is clearly linear. Since

$$
\begin{aligned}
\left\|F_{m p}\left\{x_{n}\right\}\right\|_{Y^{* *}} & =\left\|\sum_{n=0}^{p} f_{m n}\left(x_{n}\right)\right\|_{Y^{* *}} \leqq \sum_{n=0}^{p}\left\|f_{m n}\right\|_{B\left[X, Y^{* *}\right]}\left\|x_{n}\right\|_{X} \\
& \leqq\left(\sum_{n=0}^{p}\left\|f_{m n}\right\|_{B\left[X, Y^{* *}\right]}\right)\left\|\left\{x_{n}\right\}\right\|_{\chi},
\end{aligned}
$$

$F_{m p}$ is bounded. Now, if $\left\{x_{n}\right\}$ is a fixed convergent sequence, since $\mathfrak{T C}$ is convergence preserving, $F_{m p}\left\{x_{n}\right\}$ converges in $Y^{* *}$ as $p \rightarrow \infty$. Call the limit $F_{m}\left\{x_{n}\right\} . F_{m}$ is linear, and by the uniform boundedness principle there exist numbers $K_{m}$ independent of $p$ such that $\left\|F_{m p}\right\|_{B\left[\chi, Y^{* *}\right]} \leqq K_{m}$. Therefore, $F_{m} \in B\left[\chi, Y^{* *}\right]$ and $\left\|F_{m}\right\|_{B\left[\chi, Y^{* *}\right]} \leqq K_{m}$. Again, since $\mathscr{T}$ is convergence preserving, $y_{m}=F_{m}\left\{x_{n}\right\}$ converges in $Y^{* *}$ as $m \rightarrow \infty$. Call this limit $\mathscr{L}\left\{x_{n}\right\} . \mathcal{L}$ is clearly linear. By the uniform boundedness principle there exists a number $M$ such that $\left\|F_{m}\right\|_{B\left[\chi, Y^{* *}\right]} \leqq M$ for all $m$. Thus $\mathscr{L} \in B\left[\chi, Y^{* *}\right]$ and $\|\mathfrak{L}\|_{B\left[X_{1}, Y^{* *}\right]} \leqq M$. The statement that $\left\|F_{m}\right\|_{B\left[x, Y^{* *}\right]} \leqq M$ says that $\left\|\sum_{n=0}^{\infty} f_{m n}\left(x_{n}\right)\right\|_{Y^{* *}}$ $\leqq M \sup _{n}\left\|x_{n}\right\|_{X}$, i.e., $\left\|\sum_{n=0}^{\infty} f_{m n}\left(x_{n}\right)\right\|_{Y^{+}} \leqq M \sup _{n}\left\|x_{n}\right\|_{X}$, which is Condition A. We may also observe at this point that if we restrict $\mathscr{L}$ to constant sequences $\{x\}$ and consider the ranges of $\mathscr{L}$ and $F_{m}$ as $Y^{+}$instead of $Y^{* *}$, we have $\mathcal{L}\{x\}=\lim _{m \rightarrow \infty} F_{m}\{x\}=\lim _{m \rightarrow \infty} \sum_{n=0}^{\infty} f_{m n}(x)$ $=\lim _{m \rightarrow \infty} r_{m}(x)=L(x)$. 
3. Regular methods. As in the classical case, Conditions A, B, and $\mathrm{C}$ can be altered to give a characterization of regular summability methods. Specifically, we consider these:

Condition $\mathrm{A}^{\prime}$. There exists a number $M$ such that if $\left\{x_{n}\right\}$ is a bounded sequence of points in $X$, and $m$ is a non-negative integer, then $\left\|\sum_{n=0}^{\infty} f_{m n}\left(x_{n}\right)\right\|_{Y^{+}} \leqq M \sup _{n}\left\|x_{n}\right\|_{X}$.

Condition $\mathrm{B}^{\prime}$. If $x \in X$ and $n$ is a non-negative integer, then $f_{m n}(x)$ converges in $Y^{+}$to $\theta$ as $m \rightarrow \infty$.

Condition $\mathrm{C}^{\prime}$. If $x \in X$, then $r_{m}(x)=\sum_{n=0}^{\infty} f_{m n}(x)$ converges in $Y^{+}$ to $L(x)$ as $m \rightarrow \infty$.

It is clear that Conditions $\mathrm{B}^{\prime}$ and $\mathrm{C}^{\prime}$ imply Conditions $\mathrm{B}$ and $\mathrm{C}$.

Lemma 6. Condition A is equivalent to Condition A'.

Proof. Suppose A holds. Consider $y^{*} \in Y^{*},\left\{x_{n}\right\}$ a bounded sequence of points in $X$, and $m$ a non-negative integer. Set $\epsilon_{n}$ $=\operatorname{sgn} y^{*} f_{m n}\left(x_{n}\right)$. Then $\sum_{n=0}^{p}\left|y^{*} f_{m n}\left(x_{n}\right)\right|=\sum_{n=0}^{p} y^{*} f_{m n}\left(\epsilon_{n} x_{n}\right)$ $=y^{*} \sum_{n=0}^{p} f_{m n}\left(\epsilon_{n} x_{n}\right)=\left|y^{*} \sum_{n=0}^{p} f_{m n}\left(\epsilon_{n} x_{n}\right)\right| \leqq\left.\left\|y^{*}\right\|\right|_{Y^{*}} M \sup _{n}\left\|x_{n}\right\|_{X}$. It follows that $y^{*} \sum_{n=0}^{p} f_{m n}\left(x_{n}\right)$ converges, i.e., $\sum_{n=0}^{\infty} f_{m n}\left(x_{n}\right)$ exists in $Y^{+}$. We estimate its norm as a point in $Y^{+}$:

$$
\begin{aligned}
& \left\|\sum_{n=0}^{\infty} f_{m n}\left(x_{n}\right)\right\|_{Y^{+}} \\
& \quad=\sup _{\|y *\| \leqq 1}\left|\lim _{p \rightarrow \infty} y^{*} \sum_{n=0}^{p} f_{m n}\left(x_{n}\right)\right|=\sup _{\|y *\| \leqq 1} \lim _{p \rightarrow \infty}\left|y^{*} \sum_{n=0}^{p} f_{m n}\left(x_{n}\right)\right| \\
& \quad \leqq \sup _{\|y *\| \leqq 1} \lim _{p \rightarrow \infty}\left\|y^{*}\right\| Y_{Y^{*}}\left\|\sum_{n=0}^{p} f_{m n}\left(x_{n}\right)\right\|_{Y} \leqq \sup _{\|y *\| \leqq 1} \lim _{p \rightarrow \infty}\left\|y^{*}\right\|_{Y^{*}} M \sup _{n}\left\|x_{n}\right\|_{X} \\
& \quad \leqq M \sup _{n}\left\|x_{n}\right\|_{X} .
\end{aligned}
$$

This is Condition $\mathrm{A}^{\prime}$.

Now suppose that $A^{\prime}$ holds and $\left\{x_{n}\right\}$ is a bounded sequence of points in $X$. Consider a new sequence $\left\{x_{n}^{\prime}\right\}$ defined by $x_{n}^{\prime}=x_{n}$ if $n \leqq p$ and $x_{n}^{\prime}=\theta$ if $n>p$. Then

$$
\begin{aligned}
M \sup _{n}\left\|x_{n}\right\|_{X} & \geqq M \sup _{n}\left\|x_{n}^{\prime}\right\|_{X} \geqq\left\|\sum_{n=0}^{\infty} f_{m n}\left(x_{n}^{\prime}\right)\right\|_{Y^{+}}=\left\|\sum_{n=0}^{p} f_{m n}\left(x_{n}^{\prime}\right)\right\|_{Y^{+}} \\
& =\left\|\sum_{n=0}^{p} f_{m n}\left(x_{n}^{\prime}\right)\right\|_{Y}=\left\|\sum_{n=0}^{p} f_{m n}\left(x_{n}\right)\right\|_{Y} \cdot
\end{aligned}
$$

This is Condition A.

Observe that if Condition A holds and $\left\{x_{n}\right\}$ is a bounded sequence 
of points in $X$, the proof of Lemma 6 shows that the infinite sum in Condition $\mathrm{A}^{\prime}$ exists. If we take $\left\{x_{n}\right\}=\{x\}$, it shows that the infinite sums in Conditions $\mathrm{C}$ and $\mathrm{C}^{\prime}$ exist. We state this formally as a lemma.

LEMMA 7. Either of the Conditions A or $\mathrm{A}^{\prime}$ imply that $\sum_{n=0}^{\infty} f_{m n}\left(x_{n}\right)$ exists in $Y^{+}$for any bounded sequence $\left\{x_{n}\right\}$ of points in $X$.

THEOREM 2. If $X$ is complete, then $\mathfrak{T}$ is regular relative to a linear transformation $L$ from $X$ to $Y^{+}$if and only if Conditions $\mathrm{A}^{\prime}, \mathrm{B}^{\prime}$, and $\mathrm{C}^{\prime}$ hold.

Proof. We have noted that Conditions $\mathrm{A}^{\prime}, \mathrm{B}^{\prime}$, and $\mathrm{C}^{\prime}$ imply Conditions $\mathrm{A}, \mathrm{B}$, and $\mathrm{C}$. In fact, $c_{n}(x)=\theta$ and $\lim _{m \rightarrow \infty} r_{m}(x)=L(x)$ in $Y^{+}$. By Theorem 1, $\mathfrak{T}$ is convergence preserving, and, since $c_{n}(x)=\theta, \mathfrak{T}$ is regular relative to $L$. Therefore, Conditions $A^{\prime}, B^{\prime}$, and $C^{\prime}$ are sufficient. Recall that Lemma 4 applies so that $L$ is a bounded transformation. The necessity of Conditions $\mathrm{A}^{\prime}, \mathrm{B}^{\prime}$, and $\mathrm{C}^{\prime}$ is proven the same as in Theorem 1 except for trivial modifications.

4. Equivalence of the Hausdorff moment problem and the Riesz representation theorem.

Definition 5. The statement that $\left\{\mu_{n}\right\}$ is a moment sequence means that the Hausdorff method generated by $\left\{\mu_{n}\right\}$ satisfies Condition A of Theorem 1.

LEMMA 8. $\left\{\mu_{n}\right\}$ is a moment sequence if and only if there exists an $M$ independent of $m$ and $x_{n}$ such that

$$
\left\|\sum_{n=0}^{m}\left(\begin{array}{l}
m \\
n
\end{array}\right) \Delta^{m-n} \mu_{n} \cdot x_{n}\right\|_{Y} \leqq M \sup _{n}\left\|x_{n}\right\|_{X}
$$

for each bounded sequence $\left\{x_{n}\right\}$ of points in $X$.

Proof. By Lemma 6, Condition A is equivalent to Condition $\mathrm{A}^{\prime}$. Applying $A^{\prime}$ to the Hausdorff method generated by $\left\{\mu_{n}\right\}$ yields

$$
\begin{aligned}
M \sup _{n}\left\|x_{n}\right\|_{X} \geqq\left\|\sum_{n=0}^{\infty} \lambda_{m n}\left(x_{n}\right)\right\|_{Y^{+}} & =\left\|\sum_{n=0}^{m}\left(\begin{array}{c}
m \\
n
\end{array}\right) \Delta^{m-n} \mu_{n} \cdot x_{n}\right\|_{Y^{+}} \\
& =\left\|\sum_{n=0}^{m}\left(\begin{array}{l}
m \\
n
\end{array}\right) \Delta^{m-n} \mu_{n} \cdot x_{n}\right\|_{Y} .
\end{aligned}
$$

Conversely, if

$$
\left\|\sum_{n=0}^{m}\left(\begin{array}{l}
m \\
n
\end{array}\right) \Delta^{m-n} \mu_{n} \cdot x_{n}\right\|_{Y} \leqq M \sup _{n}\left\|x_{n}\right\|_{X},
$$

Condition $\mathrm{A}^{\prime}$ holds and thus Condition A holds. 
We now make formal statements of the representation theorem and the moment problem.

Statement $\mathrm{S}_{1}$. If $T \in B[C, Y]$, then there exists a function $K(t)$ $\in B\left[X, Y^{+}\right]$with the $\omega$-property such that $T f=\int_{0}^{1} d K(t) \cdot f(t)[8]$.

Statement $\mathrm{S}_{2}$. If $\left\{\mu_{n}\right\}$ is a moment sequence, then there exists a $K(t) \in B\left[X, Y^{+}\right]$and satisfying the $\omega$-property such that $\mu_{n} x$ $=\int_{0}^{1} d K(t) \cdot\left(t^{n} x\right)$ for $x \in X$.

The proof showing the equivalence of $S_{1}$ and $S_{2}$ utilizes the next two lemmas about Bernstein polynomials. Slight modifications of the classical arguments will give proofs for our context.

Lemma 9. If $f \in C$, then

$$
B_{m} f(t)=\sum_{n=0}^{m}\left(\begin{array}{l}
m \\
n
\end{array}\right) t^{n}(1-t)^{m-n} f\left(\frac{n}{m}\right)
$$

converges uniformly to $f$ on $0 \leqq t \leqq 1$.

Lemma 10. If $P_{k}(t)=x_{0}+x_{1} t+\cdots+x_{k} t^{k}\left(x_{i} \in X\right)$, then $P_{k}(t) \in C$ and $P_{k}(t)=B_{m}\left(P_{k}(t)\right)-\sum_{j=1}^{k-1} p_{j k}(t) / m^{j}(k \geqq 2, n \geqq 1)$, where $p_{j k}(t)$ is a polynomial with coefficients in $X$ and is independent of $m$.

Tucker [7], [8] has shown that a given $T \in B[C, Y]$ induces a unique $\exists \in B[C R, B]$ where $C R$ denotes $C$ when $X$ is the real field, with the property that $T(f \cdot x)=(J f) \cdot x$ where $f \in C R$ and $x \in X$.

Theorem 3. If $X$ and $Y$ are complete, then Statement $\mathrm{S}_{1}$ is equivalent to Statement $\mathrm{S}_{2}$.

Proof. Assume $\mathrm{S}_{1}$ holds, and suppose that $\left\{\mu_{n}\right\}$ is a moment sequence. For $t$ real and $x \in X$, define a transformation $T$ mapping $t^{k} x$ into $\mu_{k} x$, and, similarly, define $J$ mapping $t^{k}$ into $\mu_{k}$ so that $T\left(t^{k} \cdot x\right)$ $=J\left(t^{k}\right) \cdot x=\mu_{k} x$. Define $T$ linearly so that $T\left(P_{k}\right)=T\left(\sum_{n=0}^{k} x_{n} t^{n}\right)$ $=\sum_{n=0}^{k} \mu_{n} x_{n}=\sum_{n=0}^{k} J\left(t^{n}\right) \cdot x_{n}$. We first show that $T$ is bounded as a linear transformation from $X$-valued polynomials into $Y$.

$$
\begin{aligned}
T\left(B_{m} f\right) & =\sum_{n=0}^{m}\left(\begin{array}{l}
m \\
n
\end{array}\right) T\left[t^{n}(1-t)^{m-n} f\left(\frac{n}{m}\right)\right] \\
& =\sum_{n=0}^{m}\left(\begin{array}{l}
m \\
n
\end{array}\right) J\left(t^{n}(1-t)^{m-n}\right) \cdot f\left(\frac{n}{m}\right)=\sum_{n=0}^{m}\left(\begin{array}{l}
m \\
n
\end{array}\right) \Delta^{m-n} \mu_{n} \cdot f\left(\frac{n}{m}\right) .
\end{aligned}
$$

Therefore,

$$
\begin{aligned}
\left\|T B_{m} f\right\|_{Y} & =\left\|\sum_{n=0}^{m}\left(\begin{array}{c}
m \\
n
\end{array}\right) \Delta^{m-n} \mu_{n} \cdot f\left(\frac{n}{m}\right)\right\| Y \leqq M \sup _{n}\left\|f\left(\frac{n}{m}\right)\right\|_{X} \\
& \leqq M\left\|_{f}\right\|_{C},
\end{aligned}
$$


by Lemma 8 , since $\left\{\mu_{n}\right\}$ is a moment sequence. By Lemma 10, $\left\|T\left(P_{k}\right)\right\|_{Y} \leqq\left\|T B_{m}\left(P_{k}\right)\right\|_{Y}+\sum_{j=1}^{k-1}\left(1 / m^{j}\right)\left\|T p_{j k}(t)\right\|_{Y}$, where $p_{j k}(t)$ is independent of $m$. If $\epsilon>0$, there exists $M>0$ such that $\left\|T\left(P_{k}\right)\right\|_{Y}$ $\leqq\left\|T B_{m} P_{k}\right\|_{Y}+\epsilon \leqq M\left\|P_{k}\right\|_{c}+\epsilon$. This gives the boundedness of $T$ on polynomials $P_{k}$. If, then $f \in C\left\|T\left(B_{m} f-B_{n} f\right)\right\|_{Y} \leqq M\left\|B_{m} f-B_{n} f\right\|_{c}$ implies that $\left\{T B_{m} f\right\}$ is Cauchy in $Y$, since $\left\{B_{m} f\right\}$ is Cauchy in $C$. Since $Y$ is complete, $T B_{m}(f)$ converges. Call its limit $T f . T$ is clearly linear on $C$ and $\|T(f)\|_{Y} \leqq M\left\|_{f}\right\|_{C}$. Now, by $S_{1}$, there exists $K(t)$ $\in B\left[X, Y^{+}\right]$with the $\omega$-property such that $T f=\int_{0}^{1} d K(t) \cdot f(t)$. In particular, if $f(t)=t^{n} x$, then $T\left(t^{n} x\right)=\mu_{n}(x)=\int_{0}^{1} d K(t) \cdot\left(t^{n} x\right)$.

We now show that $S_{2}$ implies $S_{1}$. Here we suppose a continuous linear mapping $T$ of $C$ into $Y$ is given. This $T$ generates a unique $J$, which maps $C R$ into $B[X, Y]$. Define $\mu_{n}=\Im\left(t^{n}\right)$. Then $J\left(t^{n}(1-t)^{m-n}\right)$ $=\Delta^{m-n} \mu_{n}$. We wish to show that $\left\{\mu_{n}\right\}$ is a moment sequence.

$$
\begin{aligned}
\left\|\sum_{n=0}^{m}\left(\begin{array}{c}
m \\
n
\end{array}\right) \Delta^{m-n} \mu_{n} \cdot x_{n}\right\|_{Y} & =\left\|\sum_{n=0}^{m}\left(\begin{array}{c}
m \\
n
\end{array}\right) J\left(t^{n}(1-t)^{m-n}\right) \cdot x_{n}\right\|_{Y} \\
& =\left\|T\left[\sum_{n=0}^{m}\left(\begin{array}{l}
m \\
n
\end{array}\right) t^{n}(1-t)^{m-n} x_{n}\right]\right\|_{Y} \\
& \leqq\|T\| \sup _{n}\left\|x_{n}\right\|_{X},
\end{aligned}
$$

the last inequality following from a theorem on convexity (see Dunford and Schwartz [1, p. 410]). By Lemma $8,\left\{\mu_{n}\right\}$ is a moment sequence. By Statement $\mathrm{S}_{2}$, there exists a $K(t) \in B\left[X, Y^{+}\right]$with the $\omega$-property such that $\mu_{n} x=\int_{0}^{1} d K(t) \cdot\left(t^{n} x\right)$. Now suppose $f \in C$. From the linearity of $\mathfrak{J}$ and the continuity of $T$, it follows that $T f$ $=\lim _{m \rightarrow \infty} T B_{m}(f)=\lim _{m \rightarrow \infty} \int_{0}^{1} d K(t) \cdot B_{m}(f)$. Gowurin [2] has shown that if $K(t)$ satisfies the $\omega$-property, and $f \in C$, then $\int_{0}^{1} d K(t) \cdot f(t)$ exists in $Y^{+}$completed. Denote this completion by $\bar{Y}^{+}$. If we consider $\lim _{m \rightarrow \infty} \int_{0}^{1} d K(t) \cdot B_{m}(f)$ as a point in this completion,

$$
\left\|\int_{0}^{1} d K(t) \cdot B_{m} f-\int_{0}^{1} d K(t) \cdot f\right\|_{\bar{Y}^{+}} \leqq\left(W_{0}^{1} K\right)\left\|B_{m} f-f\right\|_{c},
$$

so $\lim _{m \rightarrow \infty} \int_{0}^{1} d K(t) \cdot B_{m} f$ exists and is equal to $\int_{0}^{1} d K(t) \cdot f(t)$ in $\bar{Y}^{+}$. But since $T$ is continuous, $T B_{m} f$ converges to $T f$ in $Y$. Therefore the convergence of the integral $\int_{0}^{1} d K(t) \cdot B_{m}(f)$ to the integral $\int_{0}^{1} d K(t) \cdot f(t)$ actually occurs in $Y$, i.e., $T f=\int_{0}^{1} d K(t) \cdot f(t)$ in $Y$. This completes the proof of Theorem 3.

The proof of Theorem 3 provides a partial answer to a question raised by Tucker [8]: For what functions $K(t) \in B\left[X, Y^{+}\right]$and hav- 
ing the $\omega$-property will the transformation $T f=\int_{0}^{1} d K(t) \cdot f(t)$ actually map $C$ into $Y$ ?

Corollary 1. If $X$ and $Y$ are complete, a function $K(t) \in B\left[X, Y^{+}\right]$ having the $\omega$-property will generate a transformation $T \in B[C, Y]$ if and only if the sequence $\left\{\mu_{n}\right\}$ given by $\mu_{n}=J\left(t^{n}\right)=\int_{0}^{1} d K(t) \cdot t^{n}$ is a moment sequence.

In Theorem 4 and its subsequent remarks we assume $X$ and $Y$ are complete.

THEOREM 4. A Hausdorff method is convergence preserving if and only if it is generated by a moment sequence.

Proof. The proof one way is trivial because if the method is convergence preserving it must satisfy Condition $\mathrm{A}$ of Theorem 1 . This is the definition of a moment sequence. Suppose, then, that $H=\rho \mu \rho$ where $\left\{\mu_{n}\right\}$ is a moment sequence, i.e., Condition $\mathrm{A}$ is satisfied. Condition $C$ is also satisfied because

$$
\sum_{n=0}^{\infty} \lambda_{m n}(x)=\sum_{n=0}^{m}\left(\begin{array}{l}
m \\
n
\end{array}\right) \Delta^{m-n} \mu_{n} \cdot x=\mu_{0}(x),
$$

which is independent of $m$. In fact, the $L$ of Condition $C$ is $\mu_{0}$ for this case. We need only to show Condition B is satisfied, that is,

$$
\lambda_{m n}(x)=\left(\begin{array}{l}
m \\
n
\end{array}\right) \Delta^{m-n} \mu_{n} \cdot x
$$

converges in $Y^{+}$for each $n$ as $m \rightarrow \infty$. By Theorem 3, there exists $K(t) \in B\left[X, Y^{+}\right]$such that $\mu_{n} x=\int_{0}^{1} d K(t) \cdot\left(t^{n} x\right)$. If $F^{*} \in B^{*}\left[X, Y^{+}\right]$, $F^{*} K(t)$ is of bounded variation on $0 \leqq t \leqq 1$ [8]. Suppose $y^{*} \in Y^{*}$. $y^{*} \mu_{n}(x)=\int_{0}^{1} t^{n} d y^{*}(K(t) \cdot x)$. Since $y^{*}[(\cdot) x] \in B^{*}\left[X, Y^{+}\right], y^{*}[K(t) \cdot x]$ is of bounded variation on $0 \leqq t \leqq 1$. Therefore, the sequence $\left\{y^{*} \mu_{n}(x)\right\}$ is a real moment sequence and must satisfy Condition $B$ for the classical Toeplitz theorem [3], that is,

$$
\lim _{m \rightarrow \infty}\left(\begin{array}{l}
m \\
n
\end{array}\right) \Delta^{m-n}\left(y^{*} \mu_{n} x\right)
$$

converges. This may be written

$$
\lim _{m \rightarrow \infty} y^{*}\left(\begin{array}{l}
m \\
n
\end{array}\right) \Delta^{m-n} \mu_{n} x
$$

converges, which says 


$$
\lim _{m \rightarrow \infty}\left(\begin{array}{l}
m \\
n
\end{array}\right) \Delta^{m-n} \mu_{n} x
$$

exists in $Y^{+}$. This is Condition B. Since we now have all three conditions of Theorem $1, H$ is convergence preserving.

We may summarize the results of this section as follows. The following three statements about a Hausdorff method $H(\mu)$ are equivalent.

(1) $\left\{\mu_{n}\right\}$ is a moment sequence.

(2) $H(\mu)$ is convergence preserving.

(3) There exists a function $K$ such that $K(t) \in B\left[X, Y^{+}\right], K$ has the $\omega$-property, and $\mu_{n} x=\int_{0}^{1} d K(t) \cdot\left(t^{n} x\right)$ for each $x \in X$ and $n \geqq 0$.

\section{BIBLIOGRAPHY}

1. N. Dunford and J. T. Schwartz, Linear operators, Part I, Interscience, New York, 1958.

2. Mark Gowurin, Über die Stieltjesche Integration abstrakter Functionen, Fund. Math. 27 (1936), 254-268.

3. G. H. Hardy, Divergent series, Clarendon Press, Oxford, 1949.

4. T. H. Hildebrandt, On the moment problem for a finite interval, Bull. Amer. Math. Soc. 38 (1932), 269-270.

5. T. H. Hildebrandt and I. J. Schoenberg, On linear functional operations and the moment problem for a finite interval in one or several dimensions, Ann. of Math. (2) 34 (1933), 317-328.

6. F. Riesz, Sur les opérations fonctionnelles linéaires, C. R. Acad. Sci. Paris 149 (1909), 974-977.

7. D. H. Tucker, A note on the Riesz representation theorem, Proc. Amer. Math. Soc. 14 (1963), 354-358.

8. - $-A$ representation theorem for a continuous linear transformation on a space of continuous functions (to appear).

UNIVERSITY OF UTAH 